\title{
Hydrodynamical simulations of viscous overstability in Saturn's rings
}

\author{
Henrik N. Latter ${ }^{\mathrm{a}, \mathrm{b}, *}$, Gordon I. Ogilvie ${ }^{\mathrm{b}}$ \\ ${ }^{a}$ LERMA-LRA, École Normale Supérieure, 24 rue Lhomond, Paris 75005, France. \\ ${ }^{b}$ DAMTP, CMS, University of Cambridge, Wilberforce Rd, Cambridge CB3 OWA, United \\ Kingdom.
}

\begin{abstract}
We perform axisymmetric hydrodynamical simulations that describe the nonlinear outcome of the viscous overstability in dense planetary rings. These simulations are particularly relevant for Cassini observations of fine-scale structure in Saturn's A and B-ring, which take the form of periodic microstructure on the $0.1 \mathrm{~km}$ scale, and irregular larger-scale structure on 1-10 km. Nonlinear wavetrains dominate all the simulations, and we associate them with the observed periodic microstructure. The waves can undergo small chaotic fluctuations in their phase and amplitude, and may be punctuated by more formidable "wave defects' distributed on longer scales. It is unclear, however, whether the defects are connected to the irregular larger-scale variations observed by Cassini. The long-term behaviour of the simulations is dominated by the imposed boundary conditions, and more generally by the limitations of the local model we use: the shearing box. When periodic boundary conditions are imposed, the system eventually settles on a uniform travelling wave of a predictable wavelength, while reflecting boundaries, and boundaries with buffer zones, maintain a disordered state. The simulations omit self-gravity, though we examine its influence in future work.
\end{abstract}

Keywords: Planetary Rings; Saturn, Rings; Collisional Physics

\section{Introduction}

Ultraviolet and radio occultation experiments conducted by Cassini reveal that Saturn's rings exhibit axisymmetric structure on subkilometre scales (Colwell et al. 2007, Thomson et al. 2007, see also Colwell et al. 2009). This 'microstructure' takes the form of quasi-periodic variations, with wavelengths ranging between 150 and $220 \mathrm{~m}$. The prevalence of these wavelike features depends

\footnotetext{
* Corresponding author

Email addresses: henrik.latter@lra.ens.fr (Henrik N. Latter), gio10@cam.ac.uk (Gordon I. Ogilvie)
} 
on the disk's background optical thickness $\tau$. In particular, the features disappear in both very low $\tau$ areas (such as the C-ring and Cassini division) and very high $\tau$ areas (in some of the B-ring). Microstructure, as a consequence, is localised to the inner A-ring and low $\tau$ regions in the B-ring (Sremcevic et al. 2009, Colwell et al. 2009). In addition, the A and B-rings manifest interesting irregular structure on slightly longer scales, of 1-10 km (Porco et al. 2005). It remains a pressing theoretical task to explain the causes of this spontaneous pattern formation, and the relationship (if any) between the fine-scale and intermediate-scale structures observed.

Most likely these patterns are generated by a linear instability of viscous origin, the 'viscous overstability', which besets the homogeneous equilibrium of Keplerian shear. Growing modes take the form of axisymmetric density waves; by modulating the viscous stress, especially when the viscosity is an increasing function of density, such waves can extract energy from the background shear flow and become amplified rather than viscously damped. Viscous overstability has been theoretically established in hydrodynamical and kinetic models of Saturn's rings, as well as in $N$-body simulations (Schmit and Tscharnuter 1995, 1999, hereafter ST99, Spahn et al. 2000, Salo et al. 2001, Schmidt and Salo 2003, Latter and Ogilvie 2006, 2008, 2009, hereafter LO09). Some of these studies suggest that the nonlinear saturation of the instability is characterised by nonlinear travelling wavetrains (Schmidt and Salo 2003, LO09), which may in turn be punctuated by interesting 'wave defects' or modulated by larger-scale variations (LO09). On the other hand, the only published hydrodynamic simulation describes a saturation not nearly so regular (ST99), and which shows power being injected into longer and longer scales. Self-gravity also appears to be crucial, as it seems the only agent capable of halting this upward migration of power.

In this paper we make progress in understanding and synthesising these competing ideas and claims. We perform axisymmetric nonlinear hydrodynamic simulations in large shearing boxes, using an isothermal Newtonian fluid model. Different boundary conditions are utilised, so we may better interrogate the boundaries' influence on the final saturated state. Importantly, self-gravity is omitted. Self-gravity is undeniably a key player in the real rings, however its omission lets us clarify the essential dynamics of the problem and thus provide a sound base for interpreting later self-gravitating runs. These will be presented in a following paper.

In summary, we find that nonlinear wavetrains are the essential feature of all our runs (in agreement with LO09). On intermediate times, the phases and amplitudes of these waves may be subject to disordered fluctuations, and the radial domain may break up into subdomains of inward or outward propagating waves. The thin interfaces between these subdomains either generate wavetrains or are the site of colliding wavetrains. We call these wave defects 'sources' and 'shocks' respectively. On long times, however, the system's saturation is sensitive to the boundary conditions we impose. Periodic boxes, on the one hand, almost always relax into the first linearly stable uniform wavetrain, or a stable wavetrain nearby (see LO09). Reflecting boxes, on the other hand, 
witness either (a) the power of the fluid motions driven to the lengthscale of the box and to large amplitude, or (b) a shorter-wavelength disordered state in which waves are continuously generated at one boundary and swallowed by the other. The non-self-gravitating, reflecting boundary simulations of ST99 might eventually have achieved the first state, if run sufficiently long. We stress that none of this long-time behaviour is a faithful representation of the real radially structured rings. Instead, a better description is offered by the intermediate time behaviour: before the artificial influence of the boundary conditions overwhelms the dynamics. This idea is reinforced with runs employing 'buffered' periodic boundaries, which mimic the influence of the radial structure.

We conclude that the characteristic saturation of the overstability in the real rings comprises nonlinear waves of moderately fluctuating amplitude and wavelength separated by wave sources and shocks. It is natural to connect the nonlinear wavetrains to the quasiperiodic microstructure observed by Cassini, but it is unclear whether the shock/source distribution is related to the intermediatescale features on 1-10 km. The origin of this structure may, in fact, lie in a different mechanism altogether, or may be related to variations in the disk's material properties.

The paper is organised as follows. The model equations and assumptions are presented in Section 2, and the numerical set-up in Section 3. Numerical results are shown in the subsequent three sections, according to the boundary conditions used: results from periodic, reflecting, and buffered boxes can be found in Sections 4, 5 and 6, respectively. A discussion of these results, their limitations, and their relationship to the real rings of Saturn is given in Section 7 .

\section{Model equations}

In order to bring out the salient points of the nonlinear dynamics we deploy a very basic model. The planetary ring is approximated by a vertically averaged, non-self-gravitating, Newtonian fluid. In keeping with previous work, its shearing and bulk viscosities depend on surface density as power laws (Schmit and Tscharnuter 1995, Schmidt et al. 2001, LO09).

These assumptions are only rough approximations to the real rings of Saturn whose collective effects can deviate substantially from a Newtonian fluid (Latter and Ogilvie 2006, 2008). However, it permits a less cluttered picture of the key processes, before these become obscured by the complicated physics of selfgravity and granular flow. For a fuller discussion of the modelling issues, see Section 2 in LO09. On the other hand, hydrodynamical (and other continuum) models wield an advantage over $N$-body simulations because of their relatively cheap computational requirements (especially when self-gravity is involved). For one-dimensional axisymmetric simulations the computational cost is especially

low, which allows us to explore greater length and time scales than an $N$-body code can achieve at present.

We employ the shearing box formalism, a local model that approximates a 'patch' of disk anchored at a fixed radius $r_{0}$ and orbiting the central planet 
with frequency $\Omega$. The patch is represented with Cartesian geometry so that $x$ and $y$ denote the radial and azimuthal dimensions respectively (see Goldreich and Lynden-Bell 1965). The model hence neglects curvature effects and any radial stucture exhibited in the disk. However, it is finite (of radial size $L$ ) and potentially unrealistic boundary conditions must be supplied. The shearing box introduces problems of interpretation, especially in long integrations when the influence of the boundaries may be unavoidable: no matter how big we take $L$, at some very long time the boundaries could ultimately dominate the dynamics. We discuss this in more depth later in the paper. It should be stressed that though ST99 do not employ the shearing box, their 1D homogeneous cylindrical annulus is nearly the same model: our neglect of their curvature terms will only introduce relative errors of order $L / r_{0} \sim 10^{-4}$.

The governing equations are

$$
\begin{aligned}
& \partial_{t} \sigma+\partial_{i}\left(\sigma u_{i}\right)=0, \\
& \sigma\left(\partial_{t} u_{i}+u_{j} \partial_{j} u_{i}+2 \Omega \epsilon_{i z j} u_{j}\right)=-\sigma \partial_{i} \Phi_{T}-\partial_{i} P+\partial_{j} \Pi_{i j},
\end{aligned}
$$

where $\sigma, u_{i}, P, \Pi_{i j}$ are surface density, velocity, pressure, and the viscous stress respectively. The tidal potential is given by $\Phi_{T}=-3 \Omega^{2} x^{2} / 2$ and $\epsilon_{i j k}$ represents the alternating tensor. The pressure is calculated from the ideal gas equation of state,

$$
P=c^{2} \sigma,
$$

where $c$ is the constant (isothermal) sound speed. The disk scale height $H$ is defined through $H=c / \Omega$. The viscous stress is

$$
\Pi_{i j}=\nu \sigma\left(\partial_{i} u_{j}+\partial_{j} u_{i}\right)+\left(\nu_{b}-\frac{2}{3} \nu\right) \sigma\left(\partial_{k} u_{k}\right) \delta_{i j} .
$$

Finally, the kinematic shearing and bulk viscosities $\nu$ and $\nu_{b}$ are

$$
\nu=\frac{c^{2}}{\Omega} \alpha\left(\frac{\sigma}{\sigma_{0}}\right)^{\beta}, \quad \nu_{b}=\frac{c^{2}}{\Omega} \alpha_{b}\left(\frac{\sigma}{\sigma_{0}}\right)^{\beta}
$$

where $\alpha, \alpha_{b}$, and $\beta$ are dimensionless parameters, and $\sigma_{0}$ is a reference density (usually that of the homogeneous equilibrium state).

As in LO09, the values of the three viscous parameters are drawn from the $N$-body simulations of Salo et al. (2001). Full self-gravity was not employed in these runs, but its compression of the disk thickness was mimicked by increasing the vertical oscillation frequency $\Omega_{z}$ of the particles. In Table I we reproduce some of the data of these runs for different optical depths. We primarily use the parameter suites associated with $\tau=1.5$ and 2 . 


\begin{tabular}{|c|c|c|c|c|}
\hline$\tau$ & $\alpha$ & $\alpha_{b}$ & $\beta$ & $c / \Omega(\mathrm{m})$ \\
\hline \hline 0.5 & 0.348 & 1.08 & 0.67 & 2.47 \\
\hline 1.0 & 0.357 & 0.764 & 1.15 & 3.29 \\
\hline 1.5 & 0.342 & 0.681 & 1.19 & 4.42 \\
\hline 2.0 & 0.322 & 0.683 & 1.55 & 5.45 \\
\hline \hline
\end{tabular}

Table 1: Hydrodynamical parameters at different optical depths $\tau$ derived from $N$-body simulations for a disk of $1 \mathrm{~m}$ radius particles, located at a distance of $100,000 \mathrm{~km}$ from the centre of Saturn, undergoing collisions according to the Bridges et al. (1984) elasticity law, with vertical frequency enhancement of $\Omega_{z} / \Omega=3.6$ (Schmidt et al. 2001, Salo et al. 2001).

\subsection{Perturbations to Keplerian equilibrium}

By construction Eqs (1)-(2) admit the steady and homogeneous equilibrium state of Keplerian shear:

$$
\sigma=\sigma_{0}, \quad u_{x}=0, \quad u_{y}=-\frac{3}{2} \Omega x .
$$

We are interested in axisymmetric deviations from this state and therefore introduce perturbations:

$$
\sigma=\sigma_{0}+\sigma^{\prime}(x, t), \quad u_{x}=u_{x}^{\prime}(x, t), \quad u_{y}=-\frac{3}{2} \Omega x+u_{y}^{\prime}(x, t),
$$

where a prime denotes the perturbation.

After these expressions are substituted into Eqs (1)-(5), a linear stability analysis shows that the perturbations will grow exponentially when

$$
\beta>\frac{1}{3}\left(\frac{\alpha_{b}}{\alpha}-\frac{2}{3}\right) .
$$

This is the viscous overstability, according to which the unstable modes take the form of long density waves oscillating at a frequency near the epicyclic frequency (Schmit and Tscharnuter 1995, Schmidt et al. 2001, LO09). According to Table I, disks are overstable when their optical thickness is above some value between 0.5 and 1 . This indeed chimes with both the results of $N$-body simulations and instances of microstructure in Saturn's rings. However, the upper limit on microstructure recently discussed presents something of a mystery to both continuum and $N$-body models (Sremcevic et al. 2009, Colwell et al. 2009). The growth rates of the overstable modes are generally of order $\nu k^{2}$, where $k$ is the mode wavenumber. We plot the growth rate as a function of general $k$ in Figure 1 for parameters associated with $\tau=1.5$. Here the fastest growing mode has wavelength near $13 H$ (or $k H \approx 0.47$ ) and a growth rate of $0.0385 \Omega$. Wavelengths shorter than about $8.7 H$ are stable. ${ }^{1}$

\footnotetext{
${ }^{1}$ For a more complete rendition of the stability analysis the reader is referred to Schmit and Tscharnuter (1995) or LO09.
} 
We now adopt dimensions of time, space, and density that set $\Omega=1, H=1$, and $\sigma_{0}=1$. Consequently, the full nonlinear equations for finite-amplitude perturbations read as

$$
\begin{aligned}
& \partial_{t} \sigma+u_{x}^{\prime} \partial_{x} \sigma+\sigma \partial_{x} u_{x}^{\prime}=0 \\
& \partial_{t} u_{x}^{\prime}+u_{x}^{\prime} \partial_{x} u_{x}^{\prime}-2 u_{y}^{\prime}=-\frac{1}{\sigma} \partial_{x} \sigma+\frac{1}{\sigma} \partial_{x} \Pi_{x x}, \\
& \partial_{t} u_{y}^{\prime}+u_{x}^{\prime} \partial_{x} u_{y}^{\prime}+\frac{1}{2} u_{x}^{\prime}=\frac{1}{\sigma} \partial_{x} \Pi_{x y}
\end{aligned}
$$

with the viscous stress components given by

$$
\begin{aligned}
& \Pi_{x x}=\left(\alpha_{b}+\frac{4}{3} \alpha\right) \sigma^{1+\beta} \partial_{x} u_{x}^{\prime}, \\
& \Pi_{x y}=\alpha \sigma^{1+\beta}\left(-\frac{3}{2}+\partial_{x} u_{y}^{\prime}\right),
\end{aligned}
$$

and $\sigma=1+\sigma^{\prime}$. Our aim in this paper is to solve these equations numerically for the three fields $\sigma(x, t), u_{x}^{\prime}(x, t)$, and $u_{y}^{\prime}(x, t)$ on a large but finite domain $0 \leq x \leq L$, subject to various boundary conditions, initial conditions, and viscosity parameters.

\subsection{Boundary conditions}

We analyse three different boundary conditions, (a) periodic boundaries, (b) reflecting boundaries, and (c) 'buffered' periodic boundaries. Periodic boundaries force the time-dependent solution to satisfy:

$$
\sigma(0, t)=\sigma(L, t), \quad u_{x}^{\prime}(0, t)=u_{x}^{\prime}(L, t), \quad u_{y}^{\prime}(0, t)=u_{y}^{\prime}(L, t) .
$$

Reflecting boundaries, on the other hand, require

$$
u_{x}^{\prime}(0, t)=u_{x}^{\prime}(L, t)=u_{y}^{\prime}(0, t)=u_{y}^{\prime}(L, t)=0 .
$$

Both conditions conserve the total mass in the domain. In long-term integrations the influence of these boundary conditions can become crucial, especially when the elapsed time exceeds the domain crossing time of a density wave.

We also employ buffered periodic boundaries, where waves can freely leave the domain without reflecting back or reentering from the opposite boundary. Periodic boundaries are retained, but we block the transmission of information through them by incorporating 'buffer zones' that encase the domain edges. By

reducing $\beta$ to a low value in these regions any incident overstability wave will decay rapidly to zero before it reenters the domain from the opposite boundary. A conveniently simple model for $\beta$ is the 'boxcar' profile

$$
\beta=\left\{\begin{array}{l}
\beta_{0}, \quad L_{B}<x<L-L_{B}, \\
-0.5, \quad 0<x<L_{B} \text { and } L-L_{B}<x<L,
\end{array}\right.
$$


where $\beta_{0}$ is a constant value prevalent through almost all of the domain, and $L_{B}$ is the radial size of each buffer. For the parameters we use, typically $L_{B}=100$ was sufficient to prevent waves penetrating the buffers. Note that we cannot choose the lower limit of $\beta$ to be equal or below -1 , lest we instigate classical viscous instability (Lin and Bodenheimer 1981, Ward 1981, Lukkari 1981).

\subsection{Phase-space coordinates}

It can be illuminating to depict the spatio-temporal evolution of the system in terms of three phase variables that depend only on time. These define a finite-dimensional state space, or more precisely a three-dimensional projection of the infinite-dimensional function space of $\left(\sigma, u_{x}^{\prime}, u_{y}^{\prime}\right)$. In this projection, the evolution of the system is represented as a one-dimensional curve.

The three variables we use are (a) the mean kinetic energy density, (b) the rate of injection of 'epicyclic energy' by overstability, and (c) the rate of dissipation of epicyclic energy by viscosity. The kinetic energy density we define by

$$
\mathcal{K}(t)=\frac{1}{L} \int_{0}^{L} \frac{1}{2} \sigma\left[\left(u_{x}^{\prime}\right)^{2}+\left(u_{y}^{\prime}\right)^{2}\right] d x .
$$

For the rates of injection and dissipation we turn to Eq. (29) in LO09, which describes the (epicyclic) energy balance that steady nonlinear wavetrains must satisfy. From this equation we define the rate of injection by viscous overstability,

$$
\mathcal{I}(t)=\frac{1}{L} \int_{0}^{L} 6 \alpha \sigma^{1+\beta} \partial_{x} u_{y}^{\prime} d x,
$$

and the rate of viscous dissipation,

$$
\mathcal{D}(t)=\frac{1}{L} \int_{0}^{L} \sigma^{1+\beta}\left[\left(\alpha_{b}+\frac{4}{3} \alpha\right)\left(\partial_{x} u_{x}^{\prime}\right)^{2}+4 \alpha\left(\partial_{x} u_{y}^{\prime}\right)^{2}\right] d x .
$$

The steady uniform wavetrains computed in LO09 satisfy $\mathcal{D}=\mathcal{I}=$ cst, and as their kinetic energy is also constant, each wavetrain solution corresponds to a fixed point in the three-dimensional state space. In an unbounded domain, the entire family of solutions may be represented by a one-dimensional curve

$$
\mathbf{P}(\lambda)=[\mathcal{I}(\lambda), \mathcal{D}(\lambda), \mathcal{K}(\lambda)]
$$

where $\lambda$ is the wavelength of the train. In a finite periodic box, however, $\lambda$ can only take a finite number of discrete values: specifically, $\lambda=L / n$, where $n=1,2, \ldots$. In contrast, a reflecting box does not admit the wavetrains as invariant solutions, and so they cannot exist as fixed points in simulations with reflecting boundary conditions (though wavetrains can occur locally).

\section{Numerical methods}

The system of equations (8)-(12) is solved with two numerical codes: one based on a pseudospectral method, the other on centred finite differences. 


\subsection{Pseudospectral method}

A pseudospectral procedure is ideally suited for the runs utilising periodic boundary conditions, and it offers excellent accuracy. The radial dimension is partitioned into $N$ nodes, each equally spaced by $\Delta x$. The $x$-derivatives of the fields $\left(\sigma, u_{x}^{\prime}, u_{y}^{\prime}\right)$ are calculated in Fourier space using an FFT routine, while nonlinear terms are computed in real space. The Fourier transforms automatically force the solution to satisfy periodicity in $x$.

The time-stepping algorithm uses a predictor-corrector method, whereby a 5 th order Adams-Bashforth scheme accomplishes the prediction, and a 4th order Adams-Moulton scheme makes the correction. The time-step $\Delta t$ is limited by the demands of viscous diffusion, and thus must satisfy a Courant condition. For a low order method the condition is

$$
\Delta t<\frac{(\Delta x)^{2}}{2\left(\alpha_{b}+\frac{4}{3} \alpha\right)} \min _{x}\left[\sigma^{-\beta}\right] .
$$

For our higher order scheme we use this condition as a rough guide, and set $\Delta t$ equal to the right-hand side multiplied by a small 'safety factor' $F$. This we set to $F=0.1$. Note that, because $\sigma$ will depend on time, the time step will change throughout the simulation. At the resolutions we use, the restriction from wave propagation is not as strict as for diffusion, and so is not included.

Large peaks in the surface density greatly diminish the time-step via the Courant condition (19). Conversely, regions of very low density decrease the local viscosity and (potentially) force us to use a more refined spatial grid: numerical instability ensues when the (physical) viscous length falls beneath $\Delta x$ somewhere. The second issue can be managed by taking a very fine grid, of course, but this is at the expense of tightening the Courant condition. The right hand side of (19) scales like $(\Delta x)^{2}$, and so a fine grid can level a punishing constraint on the time step. In practice we eliminate the component Fourier modes of $\sigma$ with small wavelengths, keeping all modes with wavelength above (roughly) $H / 2$. This smoothing procedure averts numerical instability when a larger $\Delta x$ is adopted and keeps the size of the time-step manageable.

\subsection{Finite-difference method}

Our second numerical method uses tenth-order centred finite differences in space and a third-order explicit Runge-Kutta scheme in time. This method has nearly the accuracy of a pseudospectral algorithm but can easily cope with nonperiodic boundary conditions through the use of ghost zones. The timestep is again limited by a Courant condition related to the viscous diffusion or wave propagation across a grid zone. Our standard resolution is $0.2 H$, which is generally sufficient to capture shocks. In extremely long runs some mass loss occurs, but no significant differences were found with runs in which the density is renormalized after each timestep to maintain its average value. In a few

runs we added a small diffusivity (typically $0.01 \mathrm{cH}$ ) to the equation of mass conservation to assist with numerical stability. 
To implement reflecting boundary conditions with this method, we assign values to the ghost zones according to the desired symmetry of each variable. The velocity components are set to be odd and the density to be even with respect to the location of each boundary.

This method differs substantially from that of ST99, who used a first-order upwind scheme, typically with a resolution comparable to $H$, and captured shocks by applying an artificial viscosity to smooth variables over $3-4 H$. Our finite-difference method explicitly resolves the viscous scales on which the overstability is generated and dissipated, and does not require any artificial viscosity. A much shorter timestep is needed, however.

\subsection{Numerical tests}

Before presenting the general simulation results, we briefly demonstrate the accuracy of our numerical tools. While our two different codes, using independent numerical methods, yield simulation results in good agreement, it is also worthwhile to check their performance against established analytic or semianalytic results.

\subsubsection{Linear growth rates}

We check both codes against the linear theory of the viscous overstability in a domain with periodic boundaries. The initial condition is set to a small amplitude linear overstability mode characterised by its wavenumber $k$. The early stages of the mode's evolution, as approximated by the simulation, yield exponential growth with numerical growth rate $s$. Typically we compute $s$ after the perturbation has grown by at least two orders of magnitude. However, when $k$ is very small, $s$ must be calculated after only one order of magnitude because numerical error seeds faster growing modes after this time. By running the simulation at multiple $k \mathrm{~s}$ we can compute the numerical linear dispersion relation. Checking this against the analytic dispersion relation (Schmit and Tscharnuter 1995) furnishes a measure of how faithfully the numerical codes can reproduce the small-amplitude dynamics.

The analytic and numerical growth rates as a function of $k$ are compared in Fig. 1 for parameters associated with $\tau=1.5$ (see Table I). Here $\Delta x=0.25$, $L=256$, and $F=0.1$. The agreement is excellent, with a relative error less than $0.3 \%$. Naturally, increasing $\Delta x$ and/or $F$ worsens the relative error, though not substantially. On the other hand, decreasing these parameters does not win significantly more accuracy.

\subsubsection{Nonlinear wavetrains}

To test the nonlinear performance of the codes, we directly seed a stable steady nonlinear wavetrain, computed directly by the methods of LO09, and evolve it forward in time for a whole number of periods. The resulting profile can then be directly compared to the initial semi-analytical wave.

Once again, we take parameters associated with $\tau=1.5$, adopt periodic boundary conditions (necessarily), and use the pseudospectral code. We take a 


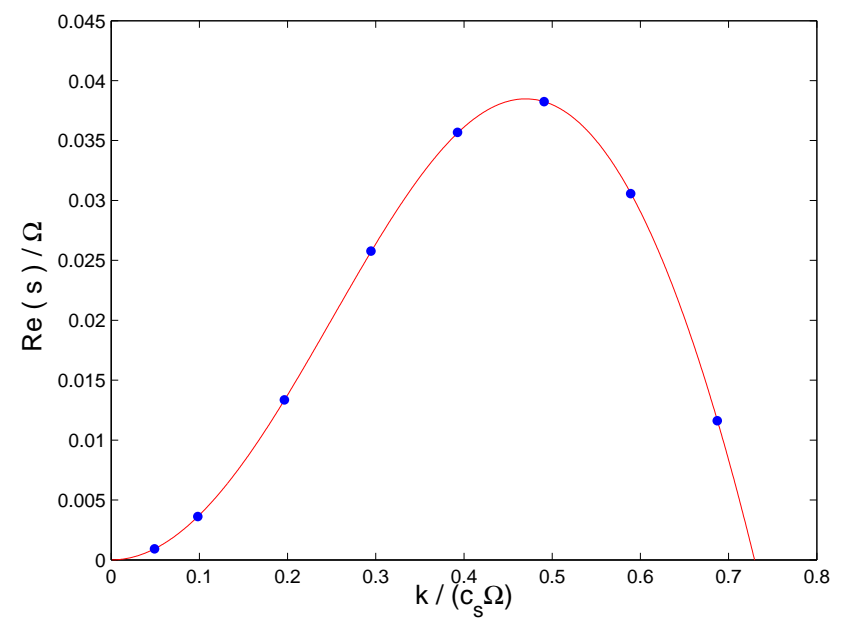

Figure 1: Linear growth rates $s$ of the overstable modes as a function of wavenumber $k$ as computed directly from the linear dispersion relation (the solid line) and numerically from the initial stages of simulations (the points). Parameters correspond to those associated with $\tau=1.5$ (see Table I), with $L=256$, and $\Delta x=0.25$ and $F=0.1$. The pseudospectral code was employed.

$\lambda=64$ wavetrain as the initial condition and evolve it for exactly 1000 periods $(\approx 993$ orbits $)$ in a box of $L=128$. The directly computed wavetrain and the numerically evolved wavetrain are presented in Fig. 2. The solid line represents the semi-analytic profile and the dots, the simulation profile. The agreement is excellent, with the kinetic energy densities $\mathcal{K}$ of the two profiles differing only at the seventh significant figure. Other choices for $\Delta x$ and $F$ yield only slightly different results.

\section{Simulation results I: Periodic boundaries}

Our first set of nonlinear results uses periodic boxes of various sizes $L$ and various viscosity parameters $\alpha, \alpha_{b}$, and $\beta$. The initial conditions are either small amplitude white noise or a small amplitude linear overstability mode of specified $k$. The main parameters we explore are associated with the $\tau=1.5$ and 2 cases (Table I), as well as the ST99 parameters, which are $\alpha=\alpha_{b}=0.262$ and $\beta=1.26$. The size of the box ranges from the small, $L=248$, to the rather large, $L=4096$. The pseudospectral code was used primarily, but its results were checked against those of the finite difference method.

\subsection{Long-time saturation: Nonlinear wavetrains}

For all parameters and box sizes the system always exhibited the same longtime saturation: a stable uniform nonlinear wavetrain. There is some variety in 

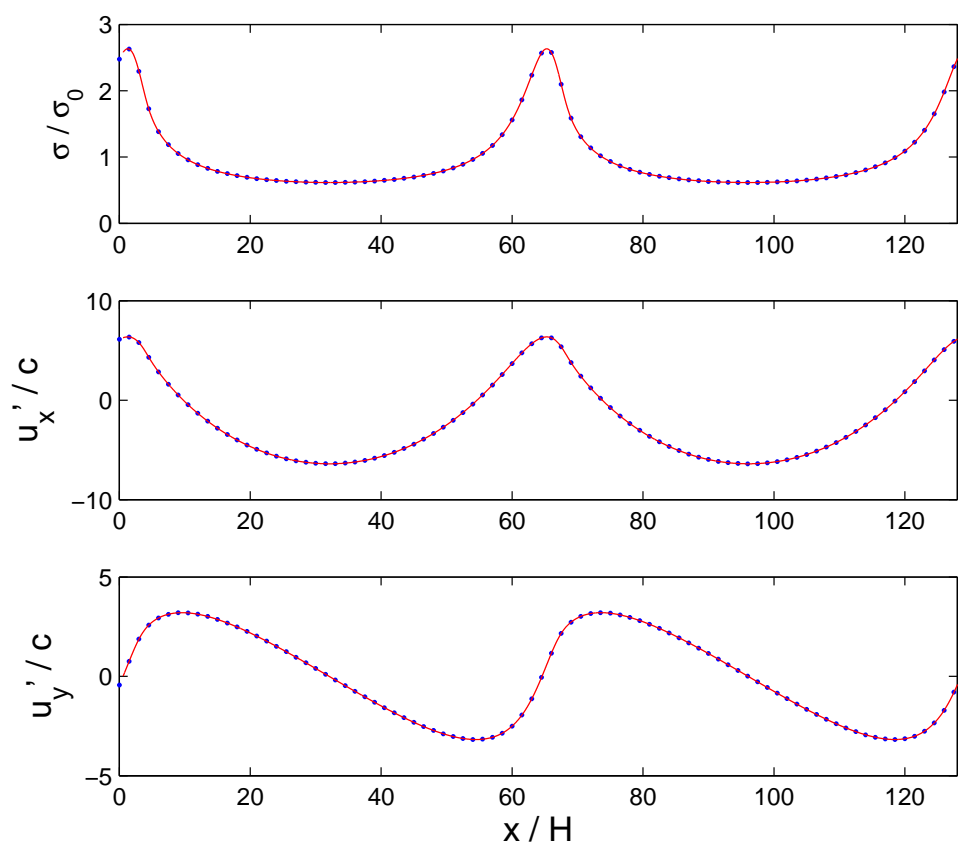

Figure 2: Two wavelengths of a steady nonlinear wavetrain as computed directly by the methods of LO09 (the solid line), and numerically by the pseudospectral code (dots). The numerical profile was computed by seeding the directly calculated wavetrain and then evolving forward for 1000 periods in a periodic box of $L=128$. Parameters chosen are associated with $\tau=1.5$, with $\Delta x=0.5, F=0.1$.

the wavelength of this solution, and the train can propagate either left or right. However, the final wavelengths are always close to the shortest linearly stable wavelength possible $\lambda_{\text {st }}$, as determined by the stability analysis of LO09. The surface density difference between the wave peaks and troughs is roughly 4 for the parameters we examine.

The variations in the final state are linked to the influence of the initial conditions. Because small deviations are exacerbated by the chaotic dynamics, small biases in the initial conditions, favouring leftward or rightward propagation or certain lengthscales, can significantly alter the state-space trajectories, leading to different final outcomes. This is more likely in larger boxes, because more stable fixed points can be supported, and as these are distributed more densely in the phase space, the basin of attraction of each is smaller.

Figures 3 and 4 show results of a simulation using ST99 parameters in a periodic box of size $L=1024$ with $\Delta x=0.25$. The kinetic energy density $(\mathcal{K})$ versus time is plotted in the former graph, and a snapshot of the state variables is plotted after 15,000 orbits in the latter. The initial conditions were 


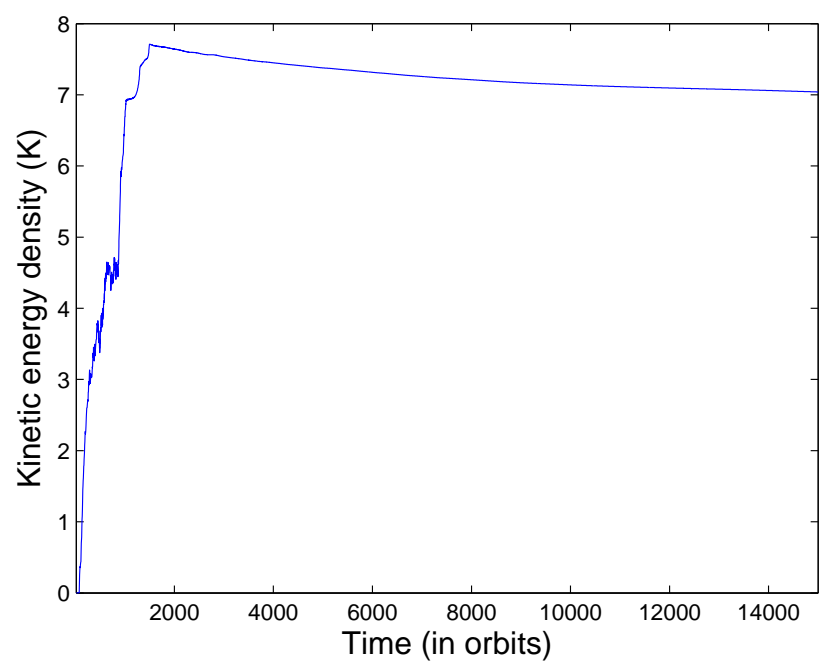

Figure 3: Kinetic energy density $\mathcal{K}$ versus time in a simulation of an overstable disk in a periodic box of $L=1024$ with $\Delta x=0.25$. The parameters correspond to those of ST99. Note that the system finally relaxes to a constant energy associated with the first stable wavetrain $\lambda=44.5$, after transient behaviour lasting roughly 2000 orbits.

low amplitude white noise. After initial transient behaviour, the disk relaxes into a constant energy configuration which is associated with the first stable wavetrain possible in the box: $\lambda=1024 / 23 \approx 44.5 .^{1}$

The final saturation achieved in the periodic boxes should be contrasted with the ST99 results with reflecting boundaries (and no self-gravity). These simulations did not yield a quasi-steady saturated state, but rather a disordered flow whereby power was continously transferred to longer and longer scales. We conclude that the boundary conditions are controlling the long-term behaviour in both simulations. In the periodic box case, the system 'senses' the translational symmetry of the domain after a sufficiently long time (the time for an overstable wave to cross the domain) and thus feels the attraction of the steady wavetrain solutions admitted by this symmetry. In contrast, the disk system in the reflecting box cannot feel these attractors because they do not exist. Instead, the disk can be drawn to large-scale standing waves fixed by the closed boundaries (see next section).

Though the final saturated state is always similar in the periodic box (nonlinear travelling waves), the intermediate time evolution, i.e. the transient behaviour, is interesting and varied, and, in fact, may be of more relevance to the evolution of the instability in the real rings of Saturn. The intermediate

\footnotetext{
${ }^{1}$ In LO09 the critical length $\lambda_{\text {st }}$ is mistakenly given as near 60 . It is in fact 44.3 .
} 

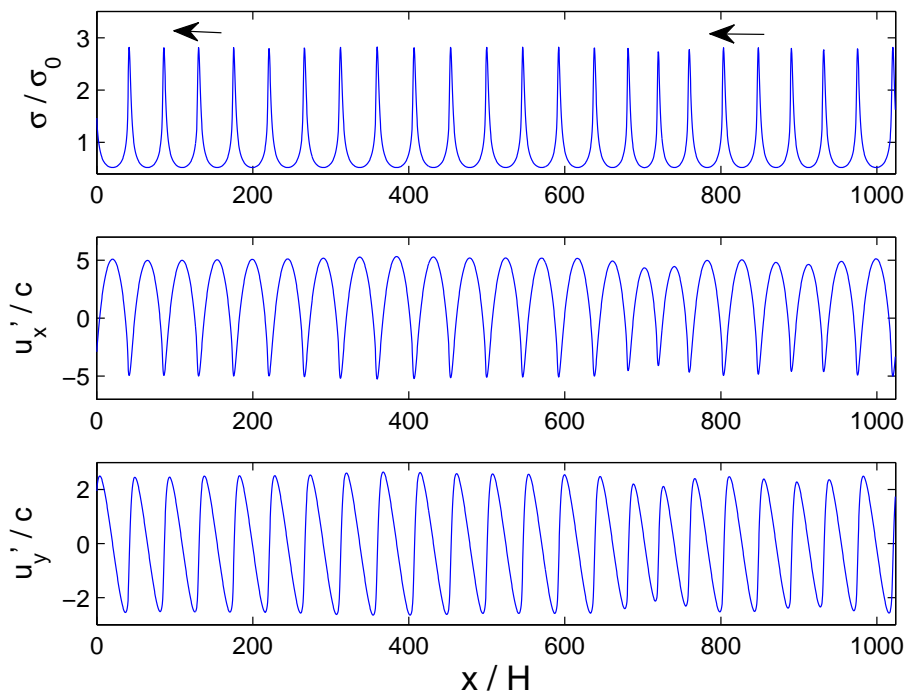

Figure 4: A snapshot of the state variables $\sigma, u_{x}^{\prime}$, and $u_{y}^{\prime}$, after 15,000 orbits. Parameters correspond to those of ST99, the box is periodic and $L=1024$. Arrows indicate the direction of propagation. Note the faint modulation in amplitude and wavenumber, which will eventually decay away.

stages exhibit phenomena presaged by LO09: the climbing of the system up the branch of nonlinear wavetrain solutions from short to longer wavelength, and the existence of shock and source structures interleaving 'patches' of nonlinear waves. We investigate each phenomenon in turn.

\subsection{Staircases}

In LO09 it is argued that the evolution of an overstable disk (in a shearing sheet) is controlled by the family of nonlinear wavetrain solutions. This branch of solutions conducts the phase space trajectories of the system away from the homogeneous state of Keplerian shear, along the unstable shorter wavelength members of the family, and towards the stable longer wavelength members. The unstable wavetrain solutions are saddle points, possessing a fast stable manifold, which attracts nearby phase trajectories, and a slow unstable manifold, which eventually repels them, thus bringing trajectories under the influence of another, longer wavelength, solution. In the infinite shearing sheet, this process will be continuous, but in the finite shearing box only a finite number of fixed points are permitted, and a discrete picture is more accurate. The evolution in this case appears something like a 'staircase', with the system jumping from one fixed point to the next until it finally comes to rest on the first stable wavetrain solution available to it. 
In this subsection this behaviour is demonstrated in a small box of $L=$ 248 , because the fixed points are fewer and better spaced, allowing a cleaner illustration. Parameters are those associated with $\tau=1.5$ and the resolution is $\Delta x=0.5$. The initial condition is set to the linear overstable mode of fastest growth with a very small amplitude. More general initial conditions, such as white noise, generate more complicated intermediate time behaviour which we present in the next subsection. We represent the staircase evolution with the time-dependent kinetic energy density $\mathcal{K}(t)$, and the three-dimensional statespace trajectories $[\mathcal{I}(t), \mathcal{D}(t), \mathcal{K}(t)]$, which one can observe bunch about the 'spine' of fixed points.

Figure 5 presents a plot of $\mathcal{K}$ as a function of time, and Figure 6 shows the phase portrait of the system's evolution as depicted in the $[\mathcal{I}, \mathcal{D}, \mathcal{K}]$ phase projection. In Fig. 5 we have labelled each 'plateau' with the wavelength of the wavetrain solution that is controlling the dynamics at that time. When resting on a plateau the system resembles the nearby wavetrain, but with some additional disorder in the train's phase and amplitude, which are the work of slow modulational instabilities. Eventually these 'secondary' instabilities drive the disk away from the solution, at which point the disk is rapidly brought within the orbit of a more energetic and longer wavelength solution.

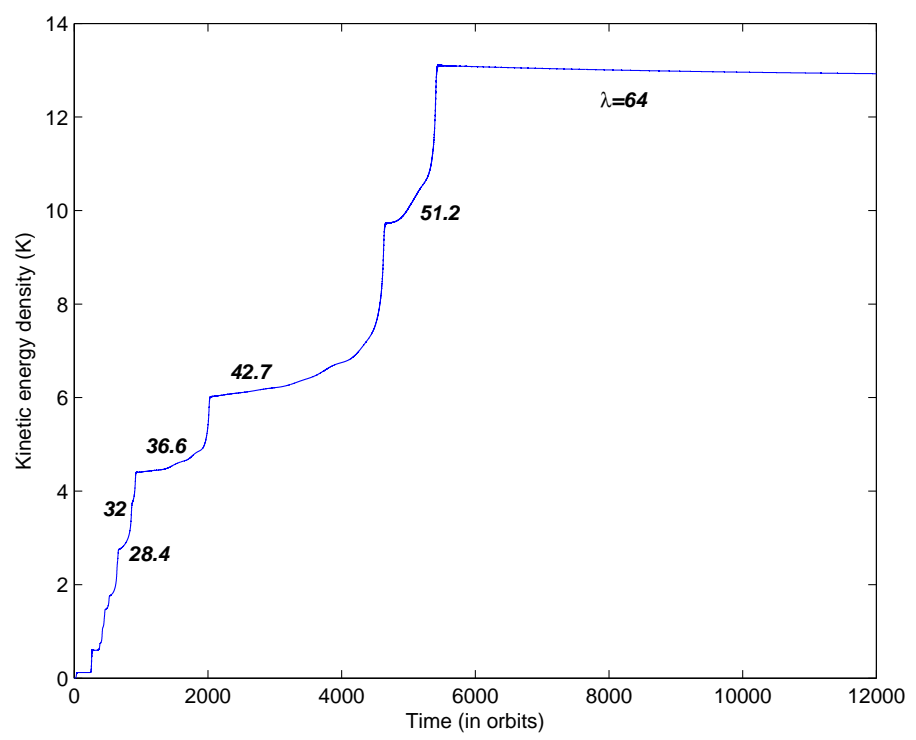

Figure 5: Kinetic energy density versus time for a simulation in a small box $L=256$ with parameters corresponding to $\tau=1.5$. The initial condition is the fastest growing linear overstability mode with small amplitude. Salient plateaus, or staircases, are labelled with the wavelength of the nearby wavetrain solution controlling the dynamics. 
This process is illustrated clearly in Fig. 6. Here the phase trajectory is represented by the blue curve, and the fixed points are denoted by red dots. The latter are calculated directly using the methods of LO09. Some of these are labelled by their wavelength. The first stable fixed point is denoted by a star, and the homogeneous state of Keplerian shear is denoted by an open circle. The system migrates from this state, which is overstable, to the first available stable state, the red star, which corresponds to a wavetrain with $\lambda=64$. The evolution follows the unstable fixed points, hopping from one to another, in dense gyres. Note that trajectories are, on average, repulsed from an unstable fixed point vertically, and attracted horizontally. The unstable manifolds are hence predominantly along the kinetic energy density coordinate $\mathcal{K}$. This makes sense because the unstable manifold is controlled by the secondary, modulational overstability which transfers energy from the background shear into the system (they are thus not 'parasitic modes', as such). This also means that the trajectories always approach the spine some distance 'above' the fixed points because they possess some residual energy in excess of the uniform wavetrains. The final approach to the stable solution is a very slow and purely vertical drop - and is dominated by the decaying modulational modes. These modes slowly remove the excess energy of the system through standard viscous dissipation. They can be observed in the faint modulation of the profiles in Fig. 4.

As the box size $L$ is increased, the number of fixed points multiplies and their relative spacing decreases. Therefore, the staircase effect becomes less and less pronounced, with the phase trajectories' ascent more continuous. On the other hand, because of the increase in the number of stable fixed points, the basin of attraction of each must shrink. Depending on the initial conditions, the system may select one of a number of final stable states. This also means the nonlinear stability of these solutions becomes more and more precarious. Conceivably, in an enormous shearing box (or infinite shearing sheet) the system may not be localised to any one solution at all. This is actually less a shortcoming of the finite shearing box model than it might appear, because a real disk is neither infinite nor radially unstructured. However, the relevance of uniform wavetrains taking up the entire domain to a real disk is also unclear. This issue we discuss in the following sections.

\subsection{Wave defects: shocks and sources}

For general small amplitude initial conditions and for larger boxes, another generic behaviour emerges in the early and intermediate stages of the evolution. Instead of a single (albeit heavily modulated) wavetrain, the domain is broken up into multiple regions of counter-propagating trains divided by one of two dynamical objects: a source or a shock. The former corresponds to a small localised region which generates waves in both directions, the latter to a region where two counter-propagating wavetrains collide without penetration. (Note that our usage of 'shock' differs from its conventional meaning in gas dynamics.) Between generation and collision, each 'patch' of wavetrain is subject to additional chaotic inhomogeneities in wavelength and amplitude. This dynamical 


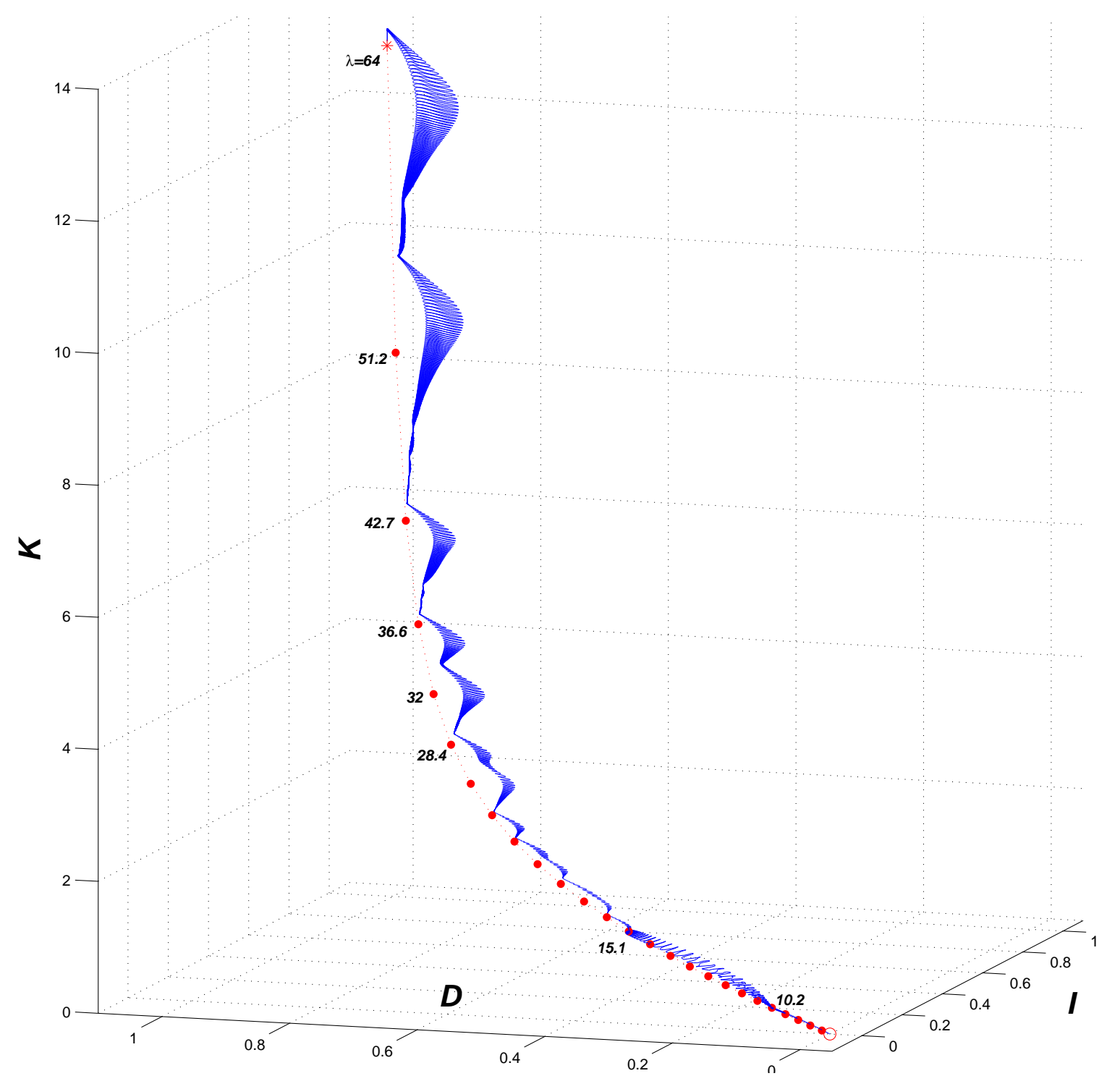

Figure 6: Phase portrait of the disk evolution described in Fig. 5. The phase space is projected onto the $3 \mathrm{D}$ grid of $(\mathcal{I}, \mathcal{D}, \mathcal{K})$. The blue curve represents the phase trajectory of the system, the red dots indicate the unstable fixed points of the system, the red star the first stable fixed point, and the open circle the homogeneous state of Keplerian shear. As is clear, the disk's evolution is guided by the family of fixed points. 
phenomenon is a natural outcome of the translational invariance of the box, and the existence of nonlinear wavetrains as argued in LO09.
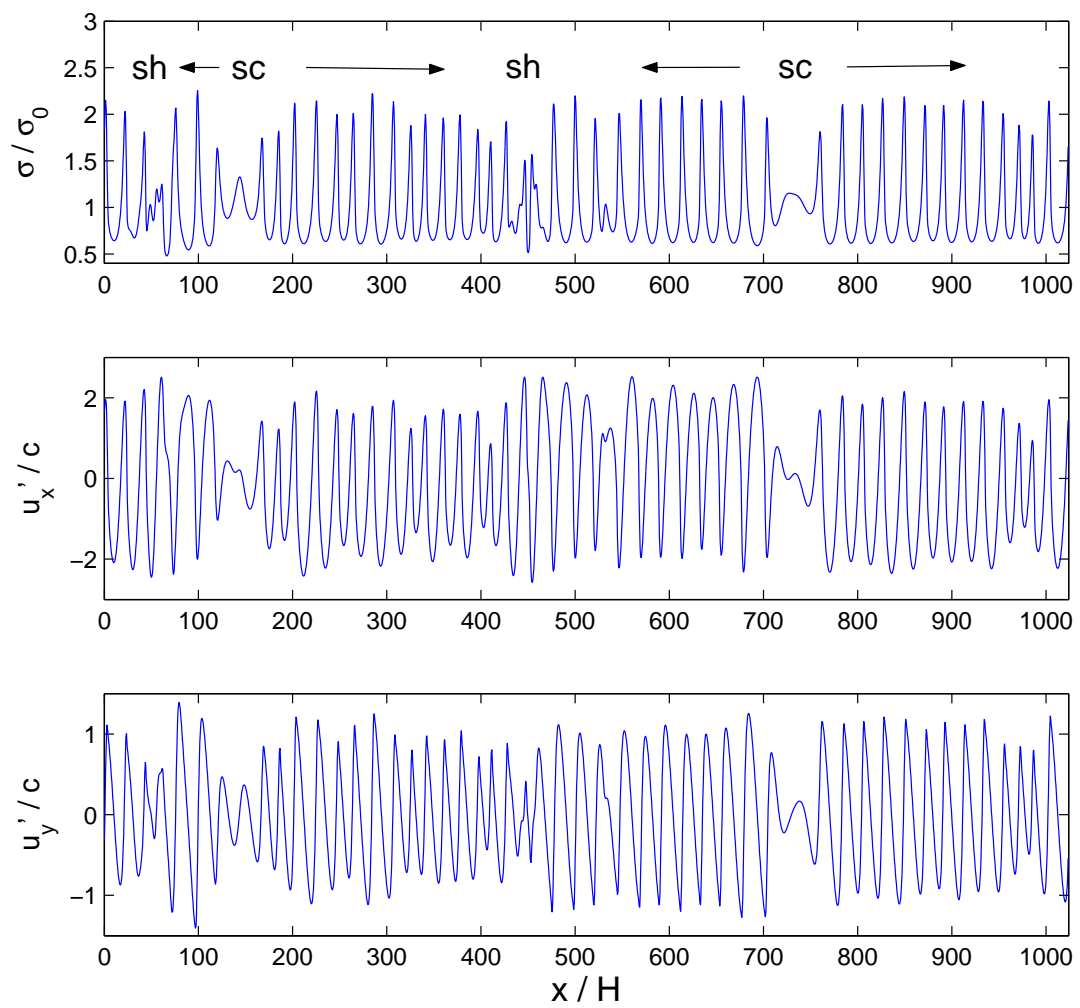

Figure 7: Snapshot of the field variables after 100 orbits in a simulation with $\tau=1.5$ parameters in a periodic box with $L=1024$ seeded with small amplitude white noise. The label 'sc' indicates a wave source and the label 'sh' a wave shock. Arrows indicate the direction of propagation of the waves.

We illustrate this behaviour with a snapshot presented in Fig. 7. Here the sources are denoted by 'sc' and the shocks by 'sh'. Arrows indicate the direction of the wave propagation. The sources correspond to relatively unperturbed regions, with $\sigma$ near 1 , and the perturbation velocities near zero. They are hence similar to the overstable homogeneous state, and thus generate linear overstability waves at a $\lambda$ near that of the fastest growing mode. This also means that there is little asymmetry in $\lambda$ about a source. As a consequence, they resemble the 'homoclons' of the complex Ginzburg-Landau equation (Aranson and Kramer 2002). This 'symmetry' contrasts with the picture described in 
LO09, where the defects separate waves of well-defined and quite different $\lambda$. Waves generally grow longer as they propagate away from sources, and are also subject to significant fluctuations and inhomogeneities.

The physical basis of these features is relatively easy to understand. In a slightly perturbed homogeneous disk, overstable modes emerge locally and form wavetrain packets. But in large domains at short times these wave packets are ignorant of what is happening in most of the box. Because different localised regions may produce waves propagating in either direction, interfaces quickly develop in order to mediate between regions that generate counterpropagating waves. These are the sources and shocks. This behaviour can be circumvented by seeding a single linear mode throughout the entire domain (as done in the previous subsection).

Notice that a source region, being relatively unperturbed, will transfer less angular momentum than its neighbouring wave-dominated regions (LO09). Consequently, a source is an impediment to the inward flow of mass: matter will tend to pile up at a source and form an overdensity. Because these regions are relatively narrow, some $50 \mathrm{H}$, the viscous timescale required to set up such a density variation is fairly short, and our simulations show an average $10 \%$ overdensity at sources once the density field is smoothed over scales of $100 H$. This variation in $\sigma$ may be observable.

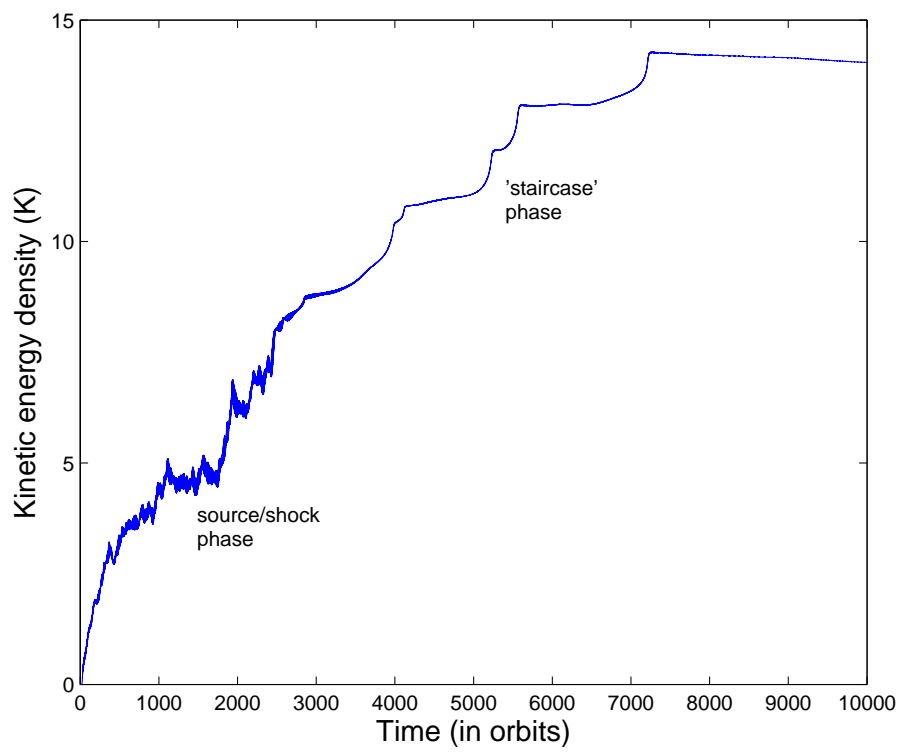

Figure 8: The kinetic energy density $\mathcal{K}$ of the system as a function of time for the simulation described in the caption of Fig. 7. Marked are the two intermediate phases, 'shock/source' and 'staircase', which the system must progress through in order to relax into a stable uniform wavetrain solution. 
In Fig. 8 we plot the kinetic energy density of the run discussed above as a function of time, with the shock/source stage marked. Note that the shock/source phase ends after a characteristic time, a few thousand orbits in this case. Shocks and sources possess their own slow dynamics and migrate through the box (cf. Section 6.2.2 in LO09). Pair by pair, the shocks and sources annihiliate each other until none are left. By orbit 2000 the last shock/source pair has disappeared and the system moves to a single disordered (and unstable) wavetrain which then undergoes the staircase process described in the last subsection.

Some of the slow shock/source dynamics are described in Fig. 9 which present a 'stroboscopic' phase-space diagram of the contours of $\sigma$ rendered in greyscale. The evolving sources correspond to the paler regions, radiating disturbances forward in time (the white diagonal lines), while the shocks correspond to regions where these disturbances meet. The plot is stroboscopic because $\sigma$ is sampled every orbit. In this way, the rapid phase propagation of the density waves of long wavelengths $2 \pi / k$ is reduced (by an amount $\Omega / k$ ) to about $(1 / 2) c H k$, which is approximately half their group velocity. The white lines hence indicate both the phase of the waves and the direction of the group velocity. At 100 orbits there exist two sources at roughly $x=150$ and $x=750$, with a shock just next to the first source near $x=50$ and another at $x=450$. The configuration at this point is plotted in Fig. 7. A little later at about 250 orbits the first shock/source pair collide near $x=0$ and then disappear. The remaining pair slowly migrate toward each other over the next thousand orbits eventually meeting and subsequently dying at around orbit 2000 .

In contrast with most regimes in the complex Ginzburg-Landau equation, the overstable disk system does not regenerate its sources and shocks (Aranson and Kramer 2002). It is unclear what drives the extinction of the defect structures. It may be because the attraction of the first stable uniform wavetrain is stronger than the attraction of the chaotic defect dynamics. This is the simplest interpretation, but it only holds in periodic domains. On the other hand, the extinction may result naturally from defects' long-distance interactions: sources and shocks might always annihilate each other because they are always attractive. This raises other questions. Do very distant wave defects interact at all? And even more crucially, are these weak dynamical interactions sensitive to self-gravity, and other physics we have omitted? The last question might be informed by the structural stability of the complex Ginzburg-Landau equation. For instance, the quintic complex Ginzburg-Landau equation, which incorporates a higher order nonlinear term, yields sources and shocks whose behaviour (and very existence, in fact) are extremely sensitive to the value of the coefficient of the new quintic term (Popp et al. 1993, Aranson and Kramer 2002). Perhaps self-gravity, or the non-Newtonian stresses in a more realistic rheological model, introduces nonlinearities which support permanent or recurring sources and shocks, by analogy with the quintic complex Ginzburg-Landau equation. 


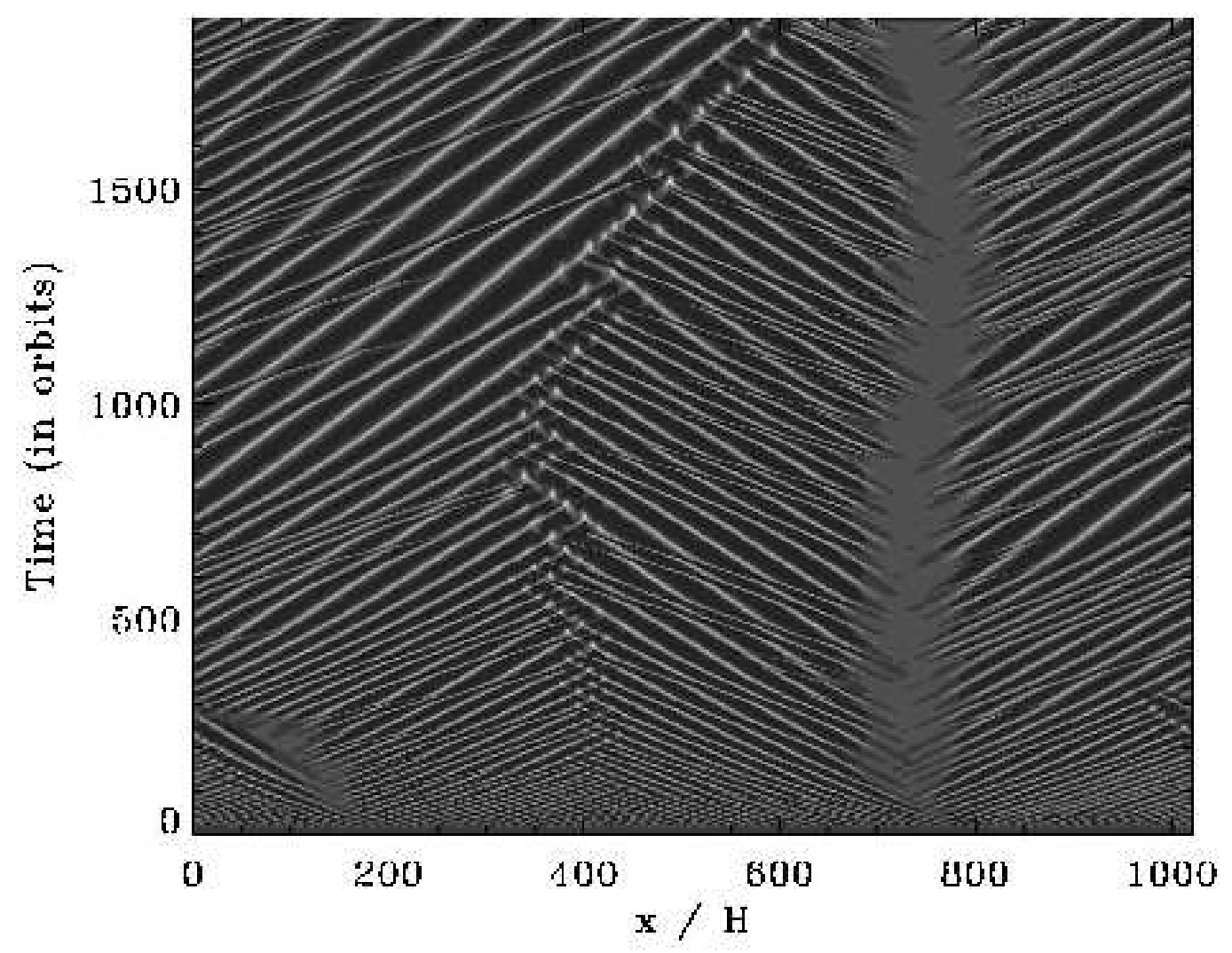

Figure 9: A 'stroboscopic' space-time diagram of the contours of $\sigma$ rendered in greyscale. White indicates density maxima (the peaks of density waves) while black represents density minima (the troughs). The grey indicates a $\sigma$ near 1 , and hence characterise wave source regions. Such regions radiate disturbances which then collide at wave shocks. Parameters are associated with $\tau=1.5$.

\subsection{Discussion}

Periodic box simulations show that at long times the disk system eventually settles on a uniform and stable travelling wavetrain: no dynamical process intervenes to prevent the system from migrating to this stable state. However, we consider this an unrealistic outcome, and an artifact of the periodic boundary conditions. Because of the long times necessary to achieve the uniform state in the simulations, the influence of the boundary conditions are inescapable. In the real rings, we expect the large-scale radial structure of the disk to exert a very 
different influence. In particular, in the real rings, steady uniform wavetrains are not exact nonlinear solutions globally; they are approximate solutions $l o-$ cally. They hence cannot function as global attractors. Instead, we expect the real system to exhibit behaviour closer to the simulational results on intermediate times, before information has fully traversed the computational box, and before the (unrealistic) global stable states completely capture the system. More specifically, we expect to observe a structure akin to the source/shock partitioning of the domain.

This idea will be explored in more detail in Section 6. Next we briefly establish the important role of the boundaries when they are assumed to be reflecting, a situation which yields similar intermediate-time behaviour to the periodic box, but an equally unrealistic long-time saturation in certain cases.

\section{Simulation results II: Reflecting boundaries}

In this section we discuss the results of numerical simulations that are similar to those described previously except that they use the reflecting boundary conditions mentioned in Section 3.2 and are carried out only with the finitedifference method. An important feature of the reflecting boundary conditions is that they do not admit solutions in the form of travelling waves, which means that the long-term evolution must be different from that described so far.

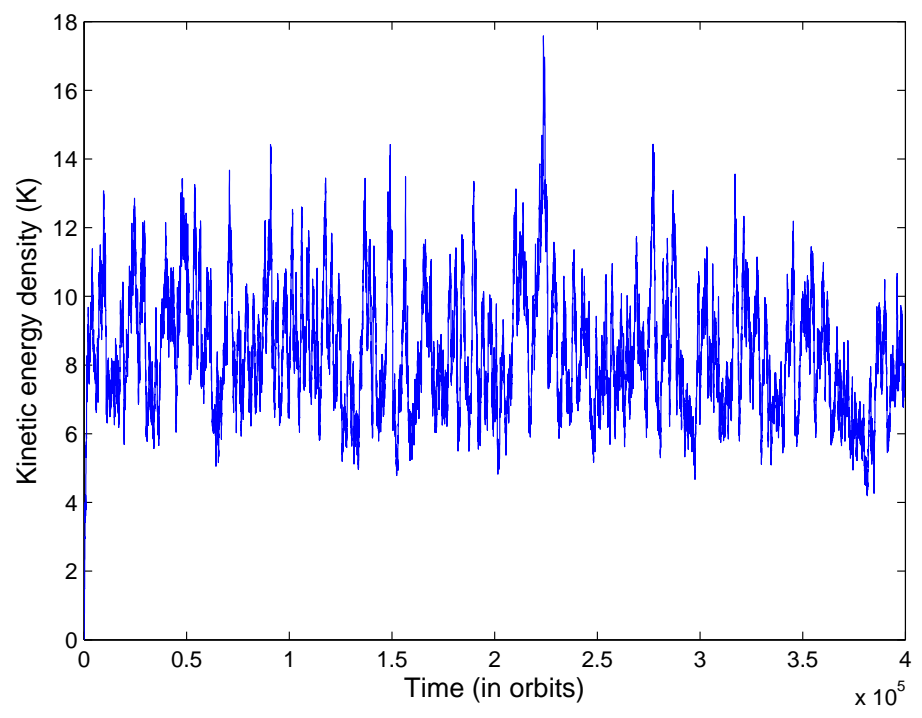

Figure 10: Kinetic energy density $\mathcal{K}$ versus time in a simulation with reflecting boundaries and viscous parameters in accordance with $\tau=1.5$. The box size is $L=1024 \mathrm{H}$. After the initial linear and shock/source phases the system settles into a statistically steady state for the length of the run, which is $4 \times 10^{5}$ orbits. 
We first consider cases with the parameters corresponding to $\tau=1.5$. Our longest run is in a box of size $1024 \mathrm{H}$ and extends for $4 \times 10^{5}$ orbits. The history of the mean kinetic energy density is shown in Fig. 10. After the phases of linear instability and shock/source evolution, the system quickly reaches a statistically steady state with a well defined mean energy and with large-amplitude fluctuations. In this state there is a single source near the left-hand boundary, which emits trains of waves in a cyclical fashion. Most of the domain is filled with nonlinear waves travelling to the right, which experience modulation and phase fluctuation as they are perturbed by the behaviour of the source. The waves are annihilated in a sink region close to the right-hand boundary.

Figure 11 is a stroboscopic space-time diagram representing the very early stages of this evolution. Until orbit 2000 there exist multiple shocks and sources, with sources localised at each boundary. After this initial stage, however, some of these defects destroy each other and the system relaxes into the long lived configuration described above.

When this experiment is repeated in a smaller box of size $512 \mathrm{H}$, however, we observe the eventual development of global standing waves. While a statistically quasi-stationary state is achieved for some time, after about $10^{5}$ orbits the system appears to develop a large-scale instability and the kinetic energy increases markedly, by more than an order of magnitude. The motion takes the form of an irregular standing wave that is clearly influenced by the specific boundary conditions adopted in this simulation. A similar run in a box of size $256 \mathrm{H}$ also appears to be strongly influenced by the boundaries, although it does not seem to develop a similarly coherent standing-wave pattern.

When the parameters are set to those adopted by ST99, we observe largescale instability and the eventual development of standing waves of large amplitude on the scale of the box, even in a domain of size $1024 \mathrm{H}$. We have not reproduced this behaviour in larger boxes, as the computational requirements of our simulations are greater than those of ST99 because we explicitly resolve the viscous length-scales. It is possible that all reflecting boxes eventually develop the global standing waves if given sufficient time, but that larger boxes have a longer phase of statistically quasi-stationary evolution. Though physically unreasonable in Saturn's A and B-ring, this phenomenon is potentially relevant to the development of global modes in narrow rings such as those of Uranus (e.g. Porco 1990), where the ring edges may reflect density waves.

\section{Simulation results III: Buffered periodic boundaries}

Finally, we present simulation results with buffered periodic boundaries, in which $\beta$ abruptly drops to small negative values within buffer zones located at the edges of the computational domain. The boundary conditions remain periodic. This configuration mimics open boundaries as far as nonlinear waves are concerned, because incident waves quickly decay to zero in the buffer zones. Mass, however, is conserved, and all the other numerical benefits of periodicity are retained (recall Section 2.2.). 


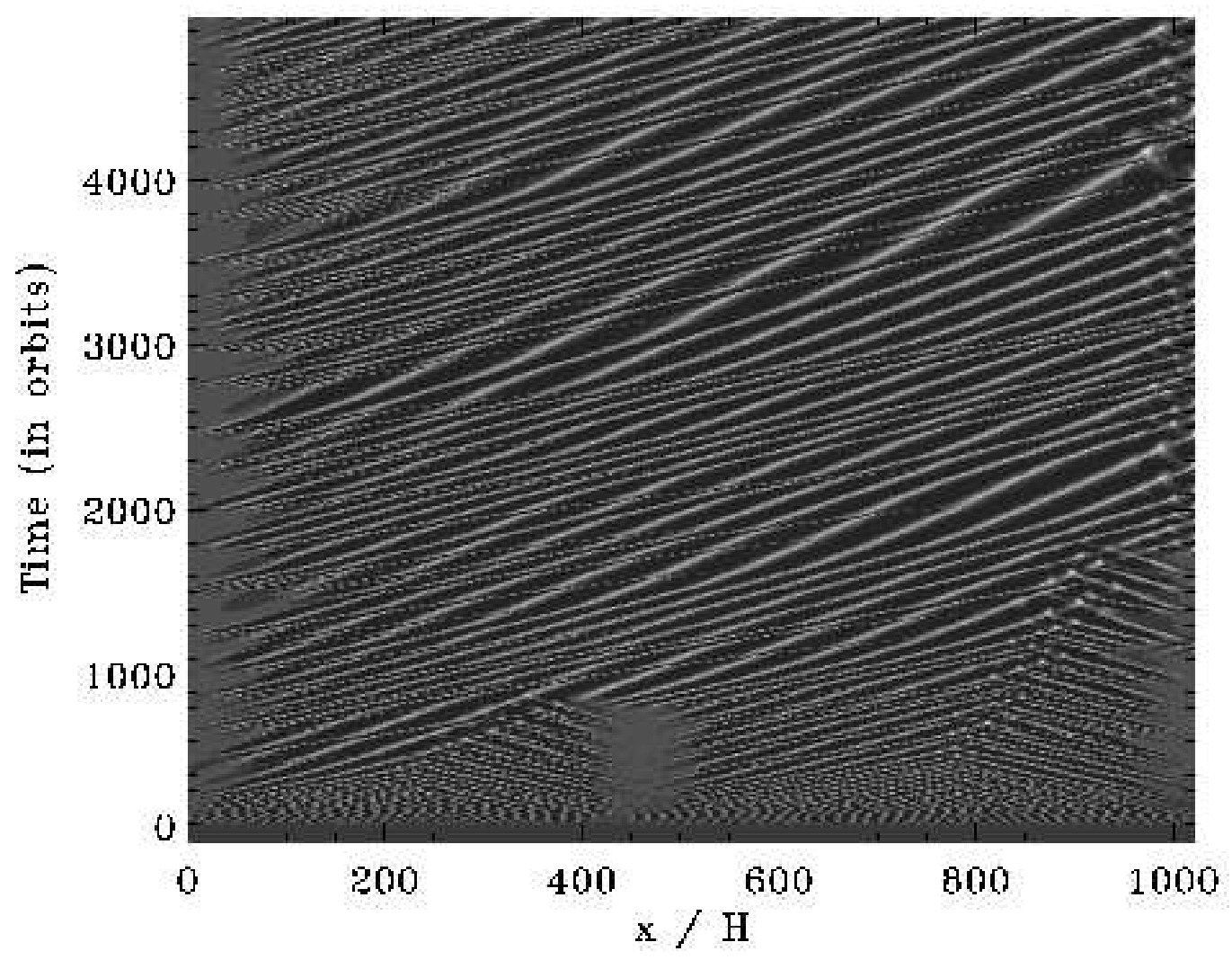

Figure 11: A space-time diagram, similar to Fig. 9, of the first 5,000 orbits of an overstable disk with reflecting boundaries. The parameters are those associated with $\tau=1.5$ and the finite-difference code is used. After a period of multiple source and shocks, the system relaxes into a long-lived configuration in which one source is localised to the left-hand boundary, and it continuously sends waves to the right boundary where they disappear.

A buffered box is a simple model that helps us understand how a real stratified patch of disk behaves. Nonlinear waves generated in the overstable region of the domain are allowed to march out of the region and be lost. They cannot 'back-react' on the region from which they emerged - an unphysical outcome of standard periodic boxes - nor are they artificially influenced by fixed reflecting boundaries. However, this also means that the domain is unphysically insulated from neighbouring patches of disk. Another important, and related, consequence of buffered boundaries is that the box can no longer support the family of exact wavetrain solutions computed in LO09 and in Section 4. The 


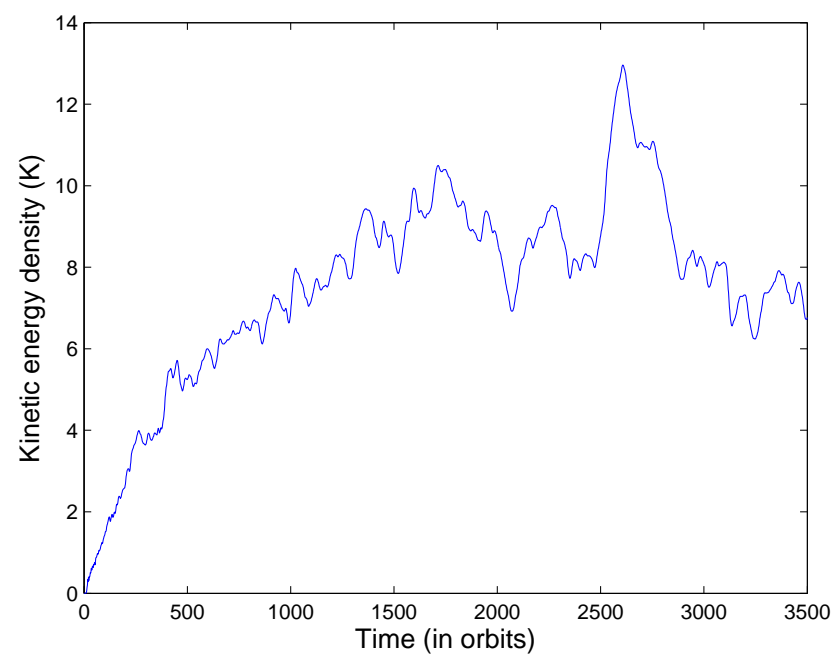

Figure 12: The kinetic energy density $\mathcal{K}$ of the system as a function of time for a simulation with buffered boundaries and parameters associated with $\tau=2$ (see Table I), and $L=1024$ and $\Delta x=0.5$. The system saturates at a fluctuating state after some 1500 orbits, once only one source remains. The buffer regions have $L_{B}=150 H$.

system must then select a different saturated state, and, on account of the domain's isolation, this must involve at least one source structure.

In Figs 12 and 13 we represent a simulation with parameters corresponding to $\tau=2$ in a buffered box of $L=1024$ with buffer zones of size 150. The first graph shows kinetic energy density against time, and the second a stroboscopic figure of surface density. The evolution described by these figures is fairly generic, and is shared by other parameter choices (such as those associated with ST99 and $\tau=1.5$ ). The system settles into a highly fluctuating state (after about 1500 orbits) characterised by a single source solution, which sends nonlinear waves through the domain into each buffer area, where they decay away. At earlier times, multiple wave defects exist. For instance, before 500 orbits the domain supports two sources and one shock dividing them. Once a shock and source pair disappear, the kinetic energy of the box increases. This is because waves can freely propagate for longer and consequently increase their wavelength and amplitude (LO09). Here the average wavelength increases from roughly $\lambda \approx 20$ to $\lambda \approx 60$ as the number of defects falls from 3 to 1 . However, far from the source (just before it collides with the far buffer) longer wavelengths can be achieved. These may be attempting to approach the first stable wavelength allowed, which for these parameters is near $120 \mathrm{H}$.

The remaining source may also migrate slowly, and we find in many simulations that lone sources will move preferentially to the edge of a buffer region. This behaviour echoes the localisation of the shocks and source in some of the 


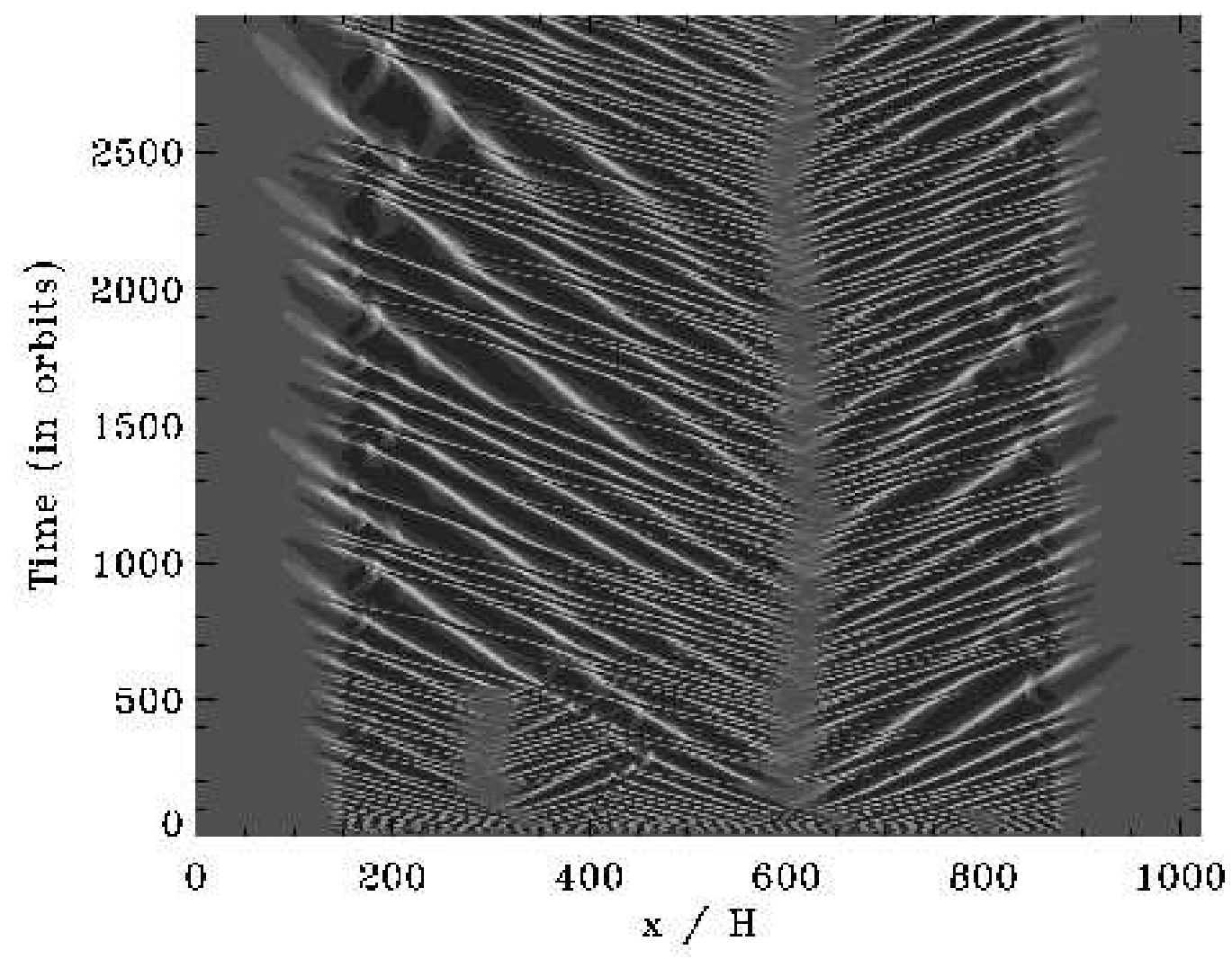

Figure 13: Stroboscopic space-time diagram of the surface density in the buffered box run described in Fig. 12. Before 500 orbits we can observe two sources and one shock between them. Near orbit 500, the source near $x=300 H$ meets the nearby shock and the two disappear. Notice that the buffer regions efficiently absorb incoming waves. Like the source regions, which are relatively unperturbed, the buffer zones are represented as pale grey.

reflecting box runs in Section 5. It also suggests that inhomogeneities in the disk's material properties could fix these structures in place.

\section{Discussion}

We now summarise the key phenomena revealed by the nonlinear simulations, specifically the nonlinear waves, the wave defects, and the influence of the boundary conditions, and we discuss their relationship with the Cassini data and the relevant modeling issues. 
All the simulations support nonlinear wavetrains at intermediate times, and almost all support wavetrains at very long times. The wavetrains may undergo low-level chaotic fluctuations in their phases and amplitudes, though at long times in periodic boxes the inhomogeneities disappear once the system settles on a globally stable uniform wavetrain. The waves yield a surface density contrast between their peaks and troughs of roughly 4 or more, and usually take wavelengths near $\lambda_{s t}$, the value of the first linearly stable wavetrain. In the buffered and fixed boundary cases, waves at shorter $\lambda$ grow longer and longer as they cross the domain so as to approach this value.

We associate these dominant features with the observations of periodic microstructure discovered by Cassini. Their wavelengths compare favourably to those observed: $\lambda_{s t} \approx 234 \mathrm{~m}$ for $\tau=1.5$, while UVIS and RSS tells us $\lambda$ lies between 150 and $220 \mathrm{~m}$ (LO09, Colwell et al. 2007, Thomson et al. 2007). On the other hand, the waves' surface density contrast between peak and trough should be sufficient to diffract the radio and UV signals in the manner observed, though this needs to be checked in detail.

The simulations also show structures on longer scales, which we term 'wavedefects'. These features divide strips of counterpropagating nonlinear waves, and take the form of either 'sources', places which generate waves, or 'shocks', places where colliding wavetrains meet. Both the shocks and sources possess their own slow dynamics and tend to wander about the computational domain. They annihilate themselves when they meet, and get stuck upon radii where the properties of the disk change abruptly - specifically, at the fixed walls or at buffer regions. In periodic domains, after a sufficiently long time, the wave defects disappear and the system moves to a steady uniform wavetrain solution. In buffered boxes, at least one source survives for the length of the run. This is also the case in reflecting boxes when global standing modes fail to develop.

Are the defect features detectable in the real rings? In particular, are they responsible for the irregular structure observed on 1-10 km scales by the Cassini cameras (Porco et al. 2005)? This is difficult to say. Originally, we argued that the observations corresponded to amplitude differences in the waves on one side of a source or shock as compared with waves on their other side (see Fig. 8 in LO09). However, the simulations show that both sets of waves around a source possess a similar wavelength initially, and thus have presumably the same photometric properties. This is also generally true for shocks. We conclude that defects probably cannot be detected by asymmetries in the waves that surround them. On the other hand, a source region typically extends for some $50 \mathrm{H}$ in radius and might be directly imaged: sources create overdensities that might be observable, and their photometric properties may differ from the adjacent wave-dominated areas. Sources are somewhat smaller than much of the observed structure, but more realistic models could yield wider sources, an issue that we intend to check. Lastly, we speculate that, given sufficient time, a source region localised to a preexisting density inhomogeneity may, in fact, exacerbate that inhomogeneity. Matter will tend to pile up at a source, fed by the waves at larger radii. In this way, a source might be indirectly responsible for sharp optical depth variations. 
As we have stressed, the long-term behaviour of the simulations depends on the boundary conditions. Periodic boundaries force the system to settle on a uniform wavetrain of a predictable wavelength, after an intermediate period characterised by sources and shocks and staircases. Reflecting boundaries yield a persistent disordered state: either the disk is drawn to large amplitude and long wavelength standing waves, or to a state whereby waves are generated near one boundary and absorbed by the other. Which of these two cases occurs depends on the choice of parameters and on the domain size. Buffered periodic boundaries eventually yield a state with only one source, often localised to a buffer edge.

Which set of simulation results best depict the real (radially structured) rings of Saturn on these scales? A perfectly uniform wavetrain solution is not a realistic outcome in the real rings, and is surely an artifact of the periodic boundaries and the neglect of radial structure. Equally, large amplitude standing waves on the box scale are unrealistic in reflecting boxes. When radial structure is mocked up by buffer zones, wave defects, which characterise intermediate term dynamics in the other cases, persist indefinitely. This suggests to us that a realistic nonlinear outcome of overstability in planetary rings involves a situation whereby shocks and source punctuate wavetrains on long scales with their radial distribution and/or slow dynamics governed by the radial structure of the disk itself.

We emphasise that additional physics may change the picture we have described. For instance, self-gravity may alter the linear stability of the global wavetrain solutions in periodic boxes, rendering all wavetrains unstable and therefore no longer attractors in periodic boxes. Alternatively, self-gravity could transform the nonlinear dynamics of the sources and shocks: instead of annihilating each other completely, they might persist indefinitely (by analogy with the structural stability of the complex Ginzburg-Landau equation). The same effects may issue not only from self gravity, but also from neglected thermodynamic and kinetic effects. If present, these effects will be in competition with the influence of the disk's radial structure, as presented in the previous paragraph.

These questions naturally lead to future work. One needs to check how self-gravity affects the axisymmetric nonlinear dynamics. Using the techniques of LO09, we intend to ascertain if self-gravity modifies the stability properties of uniform wavetrains. And then, with numerical simulations, show how the general nonlinear behaviour of the overstable disk changes. In addition, the more accurate kinetic theoretical formalism of Latter and Ogilvie (2008) will be employed in overstability simulations, as these will shed light on the dynamical importance of the rings' unusual rheological and kinetic properties. Such simulations may also directly connect with $N$-body simulations, enriching the understanding of both approaches.

Lastly, we need to investigate the non-axisymmetric element of the problem. Are the nonlinear wavetrains also unstable to disturbances with azimuthal structure (shearing waves)? Do stable nonlinear wavetrains support azimuthal wave defects in addition to radial defects? Of particular interest are the interactions between the overstability and non-axisymmetric self-gravity wakes. Under 
what circumstances do self-gravity wakes inhibit or extinguish the overstability? How do they interfere with its nonlinear saturation? Two-dimensional simulations with self-gravity may be necessary to fully assess these issues. Finally, it would also be beneficial to understand the interaction between viscous overstability and large-scale spiral waves forced by moonlets. These very long waves may themselves be overstable (Borderies et al. 1985) — what does this mean for their propagation and damping? Conversely, what is the fate of long spiral waves when they plough into a region dominated by small-scale overstability waves? Will the two processes interact destructively? In fact, recent RSS data show that the periodic microstructure peters out in the vicinity of the Pandora 5:4 density wave, an observation that might bear on this issue (Colwell et al. 2009).

\section{Acknowledgments}

The authors thank the anonymous reviewer and Frank Spahn for helpful and constructive comments. HNL thanks Jürgen Schmidt and Heikki Salo for advice and encouragement, Tobias Heinemann for his coding tips, and Pierre Lesaffre for help with IDL. This research was supported in part by STFC, Trinity College Cambridge, and the Cambridge Commonwealth Trust.

\section{References}

[1] Aranson, I. S., Kramer, L., 2002. The world of the complex GinzbergLandau equation. Reviews of Modern Physics, 74, 99-143.

[2] Borderies, N., Goldreich, P., Tremaine, S., 1985. A granular flow model for dense planetary rings. Icarus, 63, 406-420.

[3] Bridges, F., Hatzes, A., Lin, D. N. C., 1984. Structure, Stability and Evolution of Saturn's Rings. Nature, 309, 333-335.

[4] Chaté, H., 1994. Spatio-temporal intermittency regimes of the onedimensional complex Ginzburg-Landau equation. Nonlinearity, 7, 185-204.

[5] Colwell, J. E., Esposito, L. W., Sremcevic, M., Stewart, G. R., McClintock, W. E., 2007. Self-gravity wakes and radial structure of Saturn's B ring. Icarus, 190, 127-144.

[6] Colwell, J. E., Nicholson, P. D., Tiscareno M. S., Murray, C. D., French, R. G., Marouf, E. A., 2009. The Structure of Saturn's Rings. In Dougherty, M. K., Esposito, L. W., Krimigis, S. M. (eds.), Saturn from CassiniHuygens, Springer, Dordrecht Netherlands.

[7] Goldreich, P., Lynden-Bell, D., 1965. II. Spiral arms as sheared gravitational instabilities. Monthly Notices of the Royal Astronomical Society, 130, 125-158. 
[8] Hämeen-Anttila, K. A., Salo, H., 1993. Generalised Theory of Impacts in Particulate Systems. Earth, Moon, and Planets, 62, 47-84.

[9] Ipsen, M., van Hecke, M., 2001. Composite "zigzag" structures in the 1D complex Ginzburg-Landau equation. Physica D, 160, 103-115.

[10] Latter, H. N., Ogilvie, G. I., 2006. The linear stability of dilute particulate rings. Icarus, 184, 498-516.

[11] Latter, H. N., Ogilvie, G. I., 2008. Dense planetary rings and the viscous overstability. Icarus, 195, 725-751.

[12] Latter, H. N., Ogilvie, G. I., 2009. The viscous overstability, nonlinear wavetrains, and finescale structure in dense planetary rings. Icarus, 202, $565-583$.

[13] Popp, S., Stiller, O., Aranson, I., Weber, A, Kramer, L., 1993. Localized Hole Solutions and Spatiotemporal Chaos in the 1D Complex GinzburgLandau Equation. Physical Review Letters, 70, 3880-3883.

[14] Porco, C. C., 1990. Narrow rings - Observations and theory. Advances in Space Research, 10, 221-229.

[15] Porco, C. C. and 34 colleagues, 2005. Cassini Imaging Science: Initial Results on Saturn's Rings and Small Satellites. Science, 307, 1226-1236.

[16] Salo, H., Schmidt, J., Spahn, F., 2001. Viscous Overstability in Saturn's B Ring: I. Direct Simulations and Measurement of Transport Coefficients. Icarus, 153, 295-315.

[17] Schmidt, J., Salo, H., 2003. Weakly Nonlinear Model for Oscillatory Instability in Saturn's Dense Rings. Physical Review Letters, 90, 061102, 1-4.

[18] Schmidt, J., Salo, H., Spahn, F., Petzschmann, O., 2001. Viscous Overstability in Saturn's B-Ring: II. Hydrodynamic Theory and Comparison to Simulations. Icarus, 153, 316-331.

[19] Schmit, U., Tscharnuter, W. M., 1995. A Fluid Dynamical Treatment of the Common Action of Self Gravitation, Collisions and Rotation in Saturn's B-Ring. Icarus, 115, 304-319.

[20] Schmit, U., Tscharnuter, W. M., 1999. On the Formation of the Fine-Scale Structure in Saturn's B Ring. Icarus 138, 173-187.

[21] Spahn, F., Schmidt, J., Petzschmann, O., 2000. Stability Analysis of a Keplerian Disk of Granular Grains: Influence of Thermal Diffusion. Icarus, 145, 657-660.

[22] Shraiman, B. I., Pumir, A., van Saarloos, W., Hohenberg, P. C., Chaté, H., Holen, M., 1992. Spatiotemporal chaos in the one-dimensional complex Ginzburg-Landau equation. Physica D, 57, 241-248. 
[23] Sremcevic, M., Esposito, L. W., Colwell, J., 2009. Small-scale Ring Structure Observed in Cassini UVIS Occultations. AAS DPS Meeting 41, 25.03.

[24] Thomson, F S., Marouf, E. A., Tyler, G. L., French, R. G., Rappoport, N. J., 2007. Periodic microstructure in Saturn's rings A and B. Geophysical Research Letters, 34, 24, L24203. 